\title{
“UCR 55” VARIEDAD DE FRIJOL COMÚN MESOAMERICANO DE GRANO NEGRO
}

\author{
Rodolfo Araya-Villalobos ${ }^{1}$, Néstor Felipe Chaves-Barrantes ${ }^{2 / *}$, \\ Juan Carlos Hernández-Fonseca ${ }^{3}$, Carlos Cordero-Morales ${ }^{4}$ \\ Palabras clave: Andisol; antracnosis; baja fertilidad; Phaseolus vulgaris L. \\ Keywords: Andisol; anthracnose; low fertility; Phaseolus vulgaris L.
}

Recibido: 04/06/2021

RESUMEN

Introducción. La deficiencia de fósforo (P) es una de las mayores limitantes para la producción de frijol (Phaseolus vulgaris L.), en especial en las regiones tropicales. La identificación y el uso de cultivares tolerantes es esencial para reducir los costos de producción y la dependencia de los fertilizantes para suplir las necesidades de P. Objetivo. Describir el proceso de desarrollo y características agronómicas de la variedad UCR 55. Materiales y métodos. Esta variedad provino del cruzamiento NAB 44 // ROS 24 / G 13689, realizado en el Centro Internacional de Agricultura Tropical en Colombia. Fue evaluada de 1993 al 2000, por la Universidad de Costa Rica y el Ministerio de Agricultura y Ganadería. El desempeño agronómico y características de UCR 55 se determinaron con base en la evaluación de 6 viveros, 21 ensayos, 7 parcelas de validación y 5 parcelas comerciales, llevados a cabo en 15 localidades de Costa Rica. Resultados. UCR 55 es una variedad de grano negro opaco, con un peso promedio de 19,4 g en 100 semillas.

* Autor para correspondencia. Correo electrónico: nestor.chaves@ucr.ac.cr

1 Universidad de Costa Rica, Estación Experimental Agrícola Fabio Baudrit Moreno (EEAFBM), Alajuela, Costa Rica. (D) 0000-0001-8284-5856.

2 Universidad de Costa Rica, Estación Experimental Agrícola Fabio Baudrit Moreno (EEAFBM), Alajuela, Costa Rica. (D) 0000-0001-8465-8130.
Aceptado: 30/08/2021

\section{ABSTRACT}

\begin{abstract}
"UCR 55" black-seeded Mesoamerican common bean cultivar. Introduction. Phosphorus (P) deficiency is considered one of the major constraints for common bean (Phaseolus vulgaris L.) production, in particular across the tropics. Identification and use of tolerant cultivars are essential for reducing production costs and farmers' dependence on fertilizers to overcome phosphorus deficiencies. Objective. To describe the process of development and the agronomic characteristics of cultivar UCR 55. Materials and methods. This cultivar was developed from the NAB 44 // ROS 24 / G 13689 cross made at Centro Internacional de Agricultura Tropical in Colombia. It was evaluated from 1993 to 2000, by the Universidad de Costa Rica and the Ministerio de Agricultura y Ganadería. The agronomic performance and the traits of UCR 55 were determined through 6 nurseries, 21 trials, 7 validation plots and 5 commercial plots, carried out at 15 locations in Costa Rica. Results. UCR 55 is a dull black-seeded cultivar, with a mean
\end{abstract}

3 Instituto Nacional de Innovación y Transferencia en Tecnología Agropecuaria (INTA), San José, Costa Rica

(D) 0000-0002-1915-3213.

4 Instituto Nacional de Innovación y Transferencia en Tecnología Agropecuaria (INTA), Dirección Regional Central Sur, Puriscal, Costa Rica. (D) 0000-0001-9945-179X. 
En condiciones experimentales en suelos andisoles, obtuvo un rendimiento mayor a $1200 \mathrm{~kg}^{-h^{-1}}$ sin la adición de $\mathrm{P}$. El rendimiento de UCR 55 en la etapa de validación fue de una media de 1336 comparado a $1000 \mathrm{~kg} \cdot \mathrm{ha}^{-1}$ del testigo comercial Guaymí. Además, UCR 55 rindió hasta 2345 kg.ha ${ }^{-1}$ en suelos volcánicos (Fraijanes) durante esta fase. UCR 55 posee una arquitectura erecta (tipo II), resistencia (valor $\leq 3$ ) a Colletotrichum lindemuthianum y resistencia intermedia (valor s6) a Pseudocercospora griseola y a Thanatephorus cucumeris. Conclusiones. La adaptación de UCR 55 con suelos pobres en P $(<10 \mathrm{ppm})$ y su resistencia a $C$. lindemuthianum, la hacen una variedad adecuada para la siembra de frijol en zonas con una altitud superior a $1000 \mathrm{msnm}$ en Costa Rica.

\section{INTRODUCCIÓN}

El frijol (Phaseolus vulgaris L.) es un componente básico de la dieta en Costa Rica, donde el 97\% de la población lo consume (Rodríguez-González y Fernández-Rojas 2015). Su cultivo se ubica principalmente en los cantones de La Cruz, Upala, Los Chiles, Pérez Zeledón y Buenos Aires, en un rango de altitud que varía entre los 40 y $750 \mathrm{msnm}$. Su producción está principalmente en manos de pequeños productores, con explotaciones menores a las 5 ha (Hernández-Fonseca 2009b).

Los productores de frijol enfrentan varias limitaciones que afectan el rendimiento del cultivo, entre ellas los patógenos, las condiciones climáticas adversas como exceso o ausencia de precipitaciones, y altas temperaturas y problemas edáficos. En zonas bajas e intermedias, menores a $1000 \mathrm{msnm}$, las enfermedades de origen fungoso más frecuentes y problemáticas en Costa Rica son la mancha angular, causada por Pseudocercospora griseola (Sacc.) Crous \& Braun, y la mustia hilachosa, causada por Thanatephorus
100 -seed weight of $19.4 \mathrm{~g}$. Under experimental conditions in andisol soil, it yielded more than $1200 \mathrm{~kg} \cdot \mathrm{ha}^{-1}$ without phosphorus addition. The yield of UCR 55 in the validation phase was 1336 compared to $1000 \mathrm{~kg} \cdot \mathrm{ha}^{-1}$ for the commercial check Guaymí. In addition, this cultivar yielded up to $2345 \mathrm{~kg} \cdot \mathrm{ha}^{-1}$ in volcanic soils during validation. UCR 55 has an erect architecture (type II), resistance (value $\leq 3$ ) to Colletotrichum lindemuthianum and intermediate resistance (value $\leq 6)$ to Pseudocercospora griseola and Thanatephorus cucumeris. Conclusions. The adaptation to low $\mathrm{P}$ soils $(<10 \mathrm{ppm})$ and its resistance to $C$. lindemuthianum, UCR 55 is a cultivar suitable for common bean production in areas over 1000 masl in Costa Rica.

cucumeris (Frank) Dong (Hernández et al. 1999, Araya y Hernández 2003). Sobre los 1000 msnm, en zonas más frescas, se presenta Colletotrichum lindemuthianum (Sacc. \& Magn.) Scrib. (antracnosis), que puede llegar a ocasionar pérdidas hasta del 100\% (Rosas et al. 2000, Araya y Hernández 2003, Singh y Schwartz 2010). En los últimos 30 años, el nematodo Aphelenchoides besseyi Christie, causante del "amachamiento" se ha convertido un problema creciente en las zonas de producción (Chaves-Barrantes y ArayaFernández 2012, Chaves et al. 2013). Además, las larvas de los brúquidos Acanthoscelides obtectus Say y Zabrotes subfasciatus Boheman, también pueden afectar la calidad del grano y causar pérdidas considerables (hasta 35\%) durante el almacenamiento (Ishimoto y Chrispeels 1996, Miklas et al. 2006, Singh y Schwartz 2011).

Los suelos donde se siembra frijol en Costa Rica, son predominantemente ultisoles de baja fertilidad, con niveles de fósforo $(\mathrm{P})$ menores a 10 ppm (Hernández-Fonseca 2009a). Además, en zonas con altitudes iguales o mayores a $1200 \mathrm{msnm}$, en especial hacia el este y norte 
del Valle Central, en las faldas de los volcanes y algunas áreas del cantón de Coto Brus, se siembra frijol para autoconsumo y para aprovechar las podas del café (González et al. 1986, Araya y Zamora 1988). En estos lugares, predominan los suelos de tipo andisol, cuya arcilla alofana es capaz de fijar entre el 70 y $95 \%$ del fósforo (Alvarado et al. 2001).

La deficiencia de fósforo es considerada como una de las mayores limitantes para la productividad de los cultivos, en especial en los trópicos y subtrópicos (Ramaekers et al. 2010) y en el caso de frijol se estima que un 50\% del área cultivada a nivel mundial sufre de esta problemática (Beebe et al. 2009). La manera más eficiente para aminorar el problema de fertilidad por bajo nivel de fósforo, es la aplicación de fertilizantes con alto contenido de este nutriente. Sin embargo, el costo económico y ambiental, supone poca viabilidad de esta opción para las condiciones de producción de los pequeños productores de frijol en los países en desarrollo (Beebe et al. 2006). Además, se estima que las reservas de fósforo a nivel mundial podrían acabarse en los próximos 60-80 años (Beebe et al. 2006).

La identificación y el uso de genotipos más eficientes en la adquisición y utilización del fósforo, es la mejor estrategia para la producción de frijol en suelos con bajos niveles de este nutriente (Beebe 2012, Assefa et al. 2019). También reduce la dependencia de los productores del uso de fertilizantes (Singh et al. 2003). La capacidad de explorar un mayor volumen de suelo en ambientes con niveles limitantes de fósforo, varía entre genotipos y es heredable (Mourice y Tryphone 2012). Los progresos en mejoramiento genético se han logrado con genotipos que presentan mayor cantidad de raíces basales y con ángulos de inserción más rectos, mayor abundancia de raíces adventicias y mayor densidad de raíces laterales (Lynch 2019, Camilo et al. 2021). Recientemente se han identificado genotipos mesoamericanos de grano negro tolerantes a bajo P como SEN 56 y NCB 226 (Smith et al. 2019), sin embargo, en Costa Rica la investigación con énfasis en baja fertilidad data de la década de los 90. El objetivo del presente trabajo fue describir el proceso de desarrollo y las características agronómicas de la variedad UCR 55.

\section{MATERIALES Y MÉTODOS}

La línea NJBC-20601-1-CM(71V) (UCR 55) proviene del cruzamiento triple NAB $44 / /$ ROS 24 / G 13689, realizado por el Programa de Frijol del Centro Internacional de Agricultura Tropical (CIAT), en Palmira, Valle, Colombia (N 03³0', O 76²1', 965 msnm). Fue introducida a Costa Rica en mayo de 1993, en un grupo de 45 poblaciones $\mathrm{F}_{2}$ procedentes del CIAT, para la búsqueda de tolerancia a bajo fósforo, y resistencia a T. cucumeris y C. lindemuthianum. Durante el segundo semestre de 1993, las poblaciones $\mathrm{F}_{2}$ se sembraron en la Estación Experimental Agrícola Fabio Baudrit Moreno (EEAFBM) de la Universidad de Costa Rica, localizada en Alajuela, Costa Rica y otras localidades (Tabla 1). El manejo agronómico de las poblaciones no incluyó aplicación de fungicidas y con base en el método masal de una vaina por planta, se seleccionaron 67 familias $\mathrm{F}_{3}$. 
Tabla 1. Localidades donde se evaluaron los experimentos y parcelas de validación para determinar las características agronómicas y potencial de rendimiento de la variedad de frijol común UCR 55 (Phaseolus vulgaris L.). Costa Rica. $1993-2000$.

\begin{tabular}{|c|c|c|c|c|c|}
\hline Localidad & Cantón & Provincia & Región & $\begin{array}{l}\text { Localización } \\
\text { geográfica }\end{array}$ & Altitud (msnm) \\
\hline $\mathrm{EEAFBM}^{\dagger}$ & Alajuela & Alajuela & Central & $\begin{array}{l}\text { N 1000'26" } \\
\text { O } 84^{\circ} 15^{\prime} 57^{\prime \prime}\end{array}$ & 840 \\
\hline $\mathrm{SEF}^{\dagger \dagger}$ & Alajuela & Alajuela & Central & $\begin{array}{l}\text { N } 10^{\circ} 08^{\prime} 15^{\prime \prime} \\
\text { O } 84^{\circ} 11^{\prime} 36^{\prime \prime}\end{array}$ & 1742 \\
\hline Cedral & Aserrí & San José & Central & $\begin{array}{l}\text { N } 09^{\circ} 49^{\prime} 52^{\prime \prime} \\
\text { O } 84^{\circ} 08^{\prime} 02^{\prime \prime}\end{array}$ & 1864 \\
\hline Bajo Loaiza & Mora & San José & Central & $\begin{array}{l}\text { N 0949'26" } \\
\text { O 84² } 14^{\prime} 56^{\prime \prime}\end{array}$ & 840 \\
\hline La Fila & Mora & San José & Central & $\begin{array}{l}\text { N 0951'50" } \\
\text { O } 84^{\circ} 16^{\prime} 21^{\prime \prime}\end{array}$ & 992 \\
\hline El Estero & Puriscal & San José & Central & $\begin{array}{l}\text { N } 09^{\circ} 51^{\prime} 42^{\prime \prime} \\
\text { O } 84^{\circ} 17^{\prime} 29^{\prime \prime}\end{array}$ & 1005 \\
\hline La Legua & Puriscal & San José & Central & $\begin{array}{l}\text { N 0949'23"' } \\
\text { O } 84^{\circ} 21^{\prime} 16^{\prime \prime}\end{array}$ & 1109 \\
\hline El Parque & Los Chiles & Alajuela & Huetar Norte & $\begin{array}{l}\text { N } 10^{\circ} 57^{\prime} 14^{\prime \prime} \\
\text { O } 84^{\circ} 39^{\prime} 59^{\prime \prime}\end{array}$ & 48 \\
\hline Bijagua & Upala & Alajuela & Huetar Norte & $\begin{array}{l}\text { N } 10^{\circ} 43^{\prime} 49^{\prime \prime} \\
\text { O } 85^{\circ} 03^{\prime} 23^{\prime \prime}\end{array}$ & 445 \\
\hline La Vega & San Carlos & Alajuela & Huetar Norte & $\begin{array}{l}\text { N } 10^{\circ} 25^{\prime} 20^{\prime \prime} \\
\text { O } 84^{\circ} 31^{\prime} 23^{\prime \prime}\end{array}$ & 86 \\
\hline Tujankir & Guatuso & Alajuela & Huetar Norte & $\begin{array}{l}\text { N } 10^{\circ} 45^{\prime} 57^{\prime \prime} \\
\text { O } 84^{\circ} 53^{\prime} 27^{\prime \prime}\end{array}$ & 112 \\
\hline Bijagual & Buenos Aires & Puntarenas & Brunca & $\begin{array}{l}\text { N 0903'37"' } \\
\text { O 83⒉ }\end{array}$ & 387 \\
\hline Chánguena & Buenos Aires & Puntarenas & Brunca & $\begin{array}{l}\text { N } 08^{\circ} 55^{\prime} 38^{\prime \prime} \\
\text { O } 83^{\circ} 13^{\prime} 29^{\prime \prime}\end{array}$ & 462 \\
\hline Concepción & Buenos Aires & Puntarenas & Brunca & $\begin{array}{l}\text { N } 09^{\circ} 06^{\prime} 04^{\prime \prime} \\
\text { O } 83^{\circ} 28^{\prime} 06^{\prime \prime}\end{array}$ & 540 \\
\hline Veracruz & Pérez Zeledón & San José & Brunca & $\begin{array}{l}\text { N } 09^{\circ} 06^{\prime} 00^{\prime \prime} \\
\text { O 8332’37" }\end{array}$ & 615 \\
\hline
\end{tabular}

$\dagger$ Estación Experimental Agrícola Fabio Baudrit Moreno. Universidad de Costa Rica.

$\dagger$ Sub Estación Fraijanes. Universidad de Costa Rica.

En el primer semestre de 1994 se evaluaron las 67 familias $F_{3}$ en El Estero de Puriscal $y$ en el segundo semestre en la EEAFBM en Alajuela. Mediante la escogencia de una vaina por planta, se obtuvieron 375 selecciones individuales $\mathrm{F}_{4}$, que fueron evaluadas en $\mathrm{El}$ Estero de mayo a julio de 1995. De ellas, se avanzaron 10 líneas promisorias $\mathrm{F}_{5}$, que no mostraron incidencia de C. lindemuthianum y P. griseola (Tabla 2). 
Tabla 2. Líneas $\mathrm{F}_{5}$ de frijol (Phaseolus vulgaris L.) seleccionadas por su resistencia a Colletotrichum lindemuthianum (antracnosis) y a Pseudocercospora griseola (mancha angular) bajo inóculo natural. Puriscal, San José, Costa Rica. Julio, 1995.

\begin{tabular}{lll}
\hline Línea & Genealogía & Color $^{\dagger}$ \\
\hline NXBC-20594-2-CM(37V) & XAN 283 // NAB 31 // G 4017 / G 19833 & N Op \\
NHXC-20599-2-CM(64V) & XAN 283 // NAB 38 // G 4017 / G 12539 & N Op \\
NJBC-20601-1-CM(71V) & NAB 44 // ROS 24 / G 13689 & N Op \\
NJBC-20601-4-CM(55V) & NAB 44 // ROS 24 / G 13689 & N Br \\
NJBC-20604-1-CM(70V) & NAB 38 // NAB 38 / G 19833 & N Op \\
NJIC-20607-1-CM(44V) & NAB 38 / SEL1277 & N Op \\
NJIC-20607-2-CM(62V) & NAB 38 / SEL1277 & N Op \\
RXHI-20382-1-CM(70V) & NAB 38 / SEL1359 & N Op \\
NJTC-20610-2-CM(58V) & MUS 130 // XAN 275 // RAB 487 / A 247 & N Br \\
NHXC-20599-2-CM(64V) & XAN 283*(NAB 38*(G 4017*G 12539) & N Op \\
\hline
\end{tabular}

${ }^{\dagger}$ Color del grano: $\mathrm{N}=$ negro; $\mathrm{Op}=$ opaco; $\mathrm{Br}=$ brillante.

En 1996 en la Sub Estación Experimental Fraijanes (SEF) de la Universidad de Costa Rica, se evaluaron las 10 líneas promisorias $\mathrm{F}_{5}$ seleccionadas en Puriscal (Tabla 1). Se sembraron parcelas de $250 \mathrm{~m}^{2}$ en un terreno con suelo andisol y sin la adición de insumos. Se seleccionó la línea NJBC-20601-1-CM(71V), por su productividad y resistencia a $C$. lindemuthianum y $P$. griseola, y se incluyó en el Ensayo Nacional de Adaptación y Rendimiento (ENAR).

Durante 1997 y 1998, la línea NJBC20601-1-CM(71V) (UCR 55) fue evaluada en el Ensayo Nacional de Adaptación y Rendimiento (ENAR). Este experimento estuvo conformado por 16 genotipos en 1997: 12 líneas promisorias, la variedad Guaymí (Hernández-Fonseca y Araya-Villalobos 2009) como testigo nacional de grano negro, la variedad Chirripó Rojo
(Hernández-Fonseca y Araya-Villalobos 2009) como un testigo nacional de grano rojo, y 2 testigos locales, uno de grano negro y otro de grano rojo, que variaron según la localidad donde se sembró el ENAR. En 1998, se evaluaron 8 líneas promisorias, por lo que el total de genotipos evaluados fue de 12. Para el ENAR se evaluaron 10 localidades: Bijagua, Bijagual, Chánguena, Concepción, El Parque, El Estero, La Legua, La Vega, Tujankir, y Veracruz donde se sembraron un total de 17 experimentos. Se empleó un diseño de bloques completos al azar con 4 repeticiones, y la unidad experimental estuvo conformada por 6 hileras de 2,5 m de largo, separadas a $0,5 \mathrm{~m}$. La reacción de las líneas de frijol del ENAR a los patógenos, fue evaluada con la escala estándar de 1 a 9 del Centro Internacional de Agricultura Tropical (CIAT); donde 1 = sin síntomas visibles 
y 9 = síntomas de la efermedad muy severos (CIAT 1987).

En 1998, UCR 55 fue evaluada en el Vivero Histórico de Frijol Común, conformado por 31 genotipos: 7 líneas promisorias introducidas, 9 variedades comerciales liberadas antes de 1990, 5 variedades nativas de uso comercial, 5 líneas promisorias UCR y 5 variedades comerciales liberadas después de 1990. Este vivero fue sembrado en la EEAFB en Alajuela, en un terreno de baja fertilidad, con alta incidencia natural $P$. griseola, U. phaseoli, Chaetoseptoria sp., T. cucumeris y BGYMV, e inoculado con $C$. lindemuthianum. Para evaluar la reacción de los genotipos de frijol del Vivero Histórico a los patógenos, se empleó la escala estándar (CIAT 1987).

La UCR 55 se evaluó en el Vivero para Baja Fertilidad y Factores Abióticos, conformado por 49 genotipos. Se sembró en las localidades de Fraijanes (01-06-1999) y Alajuela (06-10-1999), en 2 condiciones, con y sin adición de fósforo. En Alajuela se fertilizó con 50 kg.ha- ${ }^{-1}$ de $\mathrm{P}_{2} \mathrm{O}_{5}$ y en Fraijanes con 200 kg.ha- ${ }^{-1}$ de este fertilizante. Se empleó un diseño de látice $7 \times 7$ con 3 repeticiones. En Alajuela se sembró una hilera de $5,0 \mathrm{~m}$ por genotipo y en Fraijanes 2 hileras de 2,0 m y espaciadas a 0,6 m. Se inoculó 2 veces con una suspensión de conidios de C. lindemuthianum, $\left(1 \times 10^{6}\right.$ conidios/ml) mientras que se trabajó con el inóculo natural de $P$. griseola, U. phaseoli, Chaetoseptoria sp. y BGYMV.

El 07 de octubre de 1999, se sembró también en la EEAFBM, Alajuela el Vivero de Líneas Avanzadas de Frijol (LINAF), conformado por 139 genotipos, incluido UCR 55, y que se evaluó bajo condiciones de baja fertilidad (suelo con $<10$ ppm de $\mathrm{P}$, sin adición de fertilizante en un período de 5 años y con rotaciones maíz-frijol), inoculación de C. lindemuthianum e incidencia natural de $P$. griseola, $U$. phaseoli y Chaetoseptoria sp. La unidad experimental fue una hilera de 2,0 m de largo espaciada a 0,6 $\mathrm{m}$, con una repetición por genotipo.
La fase de validación comercial de UCR 55 inició en mayo de 1999 y se sembraron parcelas en Concepción, El Estero, El Parque, Fraijanes y Veracruz. Este cultivar se evaluó junto con la variedad Bribrí (Hernández et al. 2001, Rosas et al. 2003) y los testigos nacionales Guaymí y Chirripó Rojo. Además, entre mayo y julio del 2000 se llevó a cabo una prueba comercial, en los cantones de Puriscal, Mora y Aserrí. Para ello se sembraron parcelas de $1500 \mathrm{~m}^{2}$ en las comunidades de Bajo Loaiza, Cedral, El Estero, La Fila y La Legua.

La UCR 55 fue evaluada en 15 localidades, 4 de ellas localizadas en las principales zonas de producción de frijol de Costa Rica. La altitud de los sitios de evaluación varió desde los 48 msnm en El Parque hasta los 1864 msnm en Cedral. Se cubrió todo el rango de altitud, edáfico, climático y de zonas de vida donde se ubica la producción comercial. Las áreas de siembra están localizadas en el bosque premontano muy húmedo (bmh-P) y el bosque húmedo tropical (bh-T) de acuerdo con las zonas de vida de Holdridge (1978). De 1993 al 2000, UCR 55 se incluyó en 6 viveros, 21 ensayos, 7 parcelas de validación y 5 parcelas comerciales, para determinar su resistencia a los patógenos, desempeño agronómico y potencial de rendimiento.

Los datos de rendimiento obtenidos en los distintos ensayos fueron analizados con los programas estadísticos Statistica 6.0 (StatSoft, Inc. 1984-2001) e Infostat/L (versión 2017) para determinar diferencias estadísticas a una $\mathrm{p}<0,05$, mediante un análisis combinado para las distintas épocas de siembra y localidades. Mediante observaciones de campo se registró la fenología y también se hizo una descripción de las principales características morfológicas de la variedad UCR 55, según los descriptores indicados en las guías de Muñoz et al. (1993) y Rosas et al. (2009), información fundamental para distinguirla de otras variedades. 


\section{RESULTADOS Y DISCUSIÓN}

La línea NJBC-20601-1-CM(71V) ingresó en 1993 a Costa Rica como una $\mathrm{F}_{2}$ y después de ser evaluada y avanzada en las localidades de Alajuela, Puriscal y Fraijanes. En 1996, se incluyó como $\mathrm{F}_{6}$ en el ENAR por su productividad $\mathrm{y}$ resistencia a $C$. lindemuthianum y $P$. griseola, con calificación de 3 en ambos casos, de acuerdo con la escala estándar de 1 a 9, del Centro Internacional de Agricultura Tropical (CIAT 1987). En ese año, se bautizó como UCR 55, por provenir del proyecto de selección de líneas promisorias de frijol para baja fertilidad de la Universidad de Costa Rica.

En el ENAR, evaluado durante 1997 y 1998, la UCR 55 obtuvo un rendimiento promedio de $985 \mathrm{~kg} \cdot \mathrm{ha}^{-1}$, que no difirió de los testigos nacionales de la época, Guaymí de grano negro y Chirripó Rojo de grano rojo, ni de la variedad de grano rojo Bribrí (Tabla 3), reconocida por su alto rendimiento (Hernández et al. 2001). El desempeño de UCR 55 en las regiones Brunca y Huetar Norte, principales regiones de producción de frijol en Costa Rica (Hernández-Fonseca 2009b), no difirieron de los testigos comerciales. Sin embargo, en la región Central, en la zona de Puriscal, UCR 55 aportó un rendimiento promedio superior a los testigos nacionales y a Bribrí. Además, generó una productividad estable durante los 2 años de evaluación, en contraste con la irregularidad mostrada en las localidades de las regiones Brunca y Huetar Norte. 
Tabla 3. Rendimiento promedio (kg.ha-1) de la variedad de frijol común UCR 55 (Phaseolus vulgaris L.), comparado con los testigos nacionales de grano negro (Guaymí) y rojo (Chirripó Rojo) y la variedad de grano rojo Bribrí en el Ensayo Nacional de Adaptación y Rendimiento (ENAR). Costa Rica, 1997-1998.

\begin{tabular}{|c|c|c|c|c|c|c|}
\hline \multirow{2}{*}{$\begin{array}{l}\text { Región / } \\
\text { Localidad }\end{array}$} & \multirow{2}{*}{$\begin{array}{l}\text { Época de } \\
\text { siembra }^{\dagger}\end{array}$} & \multicolumn{4}{|c|}{ Rendimiento promedio $\left(\mathrm{kg} \cdot \mathrm{ha}^{-1}\right)^{\dagger \dagger}$} & \multirow[b]{2}{*}{ Experimento } \\
\hline & & UCR 55 & Guaymí & Chirripó Rojo & Bribrí & \\
\hline \multicolumn{7}{|c|}{ Región Brunca } \\
\hline Concepción & 1997-A & $1481 \mathrm{ab}$ & $1521 \mathrm{ab}$ & $1707 \mathrm{ab}$ & $1215 \mathrm{~b}$ & 1524 \\
\hline Veracruz & 1997-A & $576 \mathrm{~b}$ & $487 \mathrm{~b}$ & $703 \mathrm{ab}$ & $582 \mathrm{~b}$ & 663 \\
\hline Bijagual & 1997-B & $957 \mathrm{a}$ & $835 \mathrm{a}$ & $725 \mathrm{a}$ & 1047 a & 872 \\
\hline Chánguena & 1997-B & $731 \mathrm{~b}$ & $692 \mathrm{~b}$ & $714 \mathrm{~b}$ & $1200 \mathrm{a}$ & 713 \\
\hline Concepción & 1997-B & $659 \mathrm{a}$ & $532 \mathrm{a}$ & $676 \mathrm{a}$ & $669 \mathrm{a}$ & 619 \\
\hline Concepción & 1998-A & $652 \mathrm{c}$ & $1327 \mathrm{ab}$ & $1232 \mathrm{ab}$ & $896 \mathrm{bc}$ & 1094 \\
\hline Veracruz & 1998-A & $824 \mathrm{a}$ & $774 \mathrm{a}$ & $322 \mathrm{~b}$ & $934 \mathrm{a}$ & 623 \\
\hline Bijagual & 1998-B & $1464 \mathrm{abc}$ & $1670 \mathrm{ab}$ & $1026 \mathrm{c}$ & $1248 \mathrm{bc}$ & 1356 \\
\hline Concepción & 1998-B & $1016 \mathrm{a}$ & 1114 a & $764 \mathrm{a}$ & $695 \mathrm{a}$ & 910 \\
\hline Promedio & & $929 \mathrm{a}$ & $995 \mathrm{a}$ & $874 \mathrm{a}$ & $943 \mathrm{a}$ & \\
\hline \multicolumn{7}{|c|}{ Región Central } \\
\hline La Legua & 1997-A & $1520 a b$ & $1560 \mathrm{a}$ & $1301 \mathrm{ab}$ & $1291 \mathrm{ab}$ & 1328 \\
\hline El Estero & 1997-B & $1275 \mathrm{a}$ & $748 \mathrm{bc}$ & $617 \mathrm{c}$ & $696 \mathrm{c}$ & 676 \\
\hline El Estero & 1998-A & 1068 a & $655 \mathrm{a}$ & $791 \mathrm{a}$ & $678 \mathrm{a}$ & 759 \\
\hline El Estero & 1998-B & $966 \mathrm{a}$ & $706 \mathrm{~b}$ & $399 \mathrm{c}$ & $746 \mathrm{~b}$ & 796 \\
\hline Promedio & & 1207 a & $917 \mathrm{bc}$ & $777 \mathrm{c}$ & $853 \mathrm{bc}$ & \\
\hline \multicolumn{7}{|c|}{ Región Huetar Norte } \\
\hline Bijagua & 1997-C & $487 \mathrm{a}$ & $553 \mathrm{a}$ & $633 \mathrm{a}$ & $528 \mathrm{a}$ & 535 \\
\hline La Vega & 1997-C & $1336 \mathrm{~b}$ & 1565 a & $1290 \mathrm{~b}$ & 1512 a & 1238 \\
\hline Tujankir & 1997-C & $693 \mathrm{a}$ & $577 \mathrm{a}$ & $697 \mathrm{a}$ & $368 \mathrm{a}$ & 564 \\
\hline El Parque & 1998-C & $1040 \mathrm{~b}$ & $1318 \mathrm{ab}$ & $941 \mathrm{~b}$ & $1310 \mathrm{ab}$ & 1178 \\
\hline Promedio & & 889 a & 1003 a & $890 \mathrm{a}$ & $930 \mathrm{a}$ & \\
\hline \multicolumn{2}{|c|}{ Promedio general } & $985 \mathrm{a}$ & $936 \mathrm{a}$ & $937 \mathrm{a}$ & 918 a & \\
\hline
\end{tabular}


En el Vivero para Baja Fertilidad y Factores Abióticos sembrado en Fraijanes, UCR 55 junto con DICTA 17, A 752, y G 11640, se evidenció el mayor rendimiento de los 49 genotipos evaluados sin la adición de fósforo, mientras que el mejor genotipo fue al que se le adicionó fósforo (Tabla 4). Alajuela UCR 55, no difirió del genotipo con mayor producción al agregarse fósforo. $\mathrm{Su}$ rendimiento fue igual al tercer mejor genotipo cuando no se agregó este nutriente. En todos los casos, el rendimiento de UCR 55 fue superior a la media del experimento (Tabla 4). Estos resultados demostraron la adaptación de la variedad UCR 55 a una condición de bajo fósforo y suelos volcánicos de tipo andisol como los de Fraijanes, localidad situada a una altitud de $1742 \mathrm{msnm}$. 


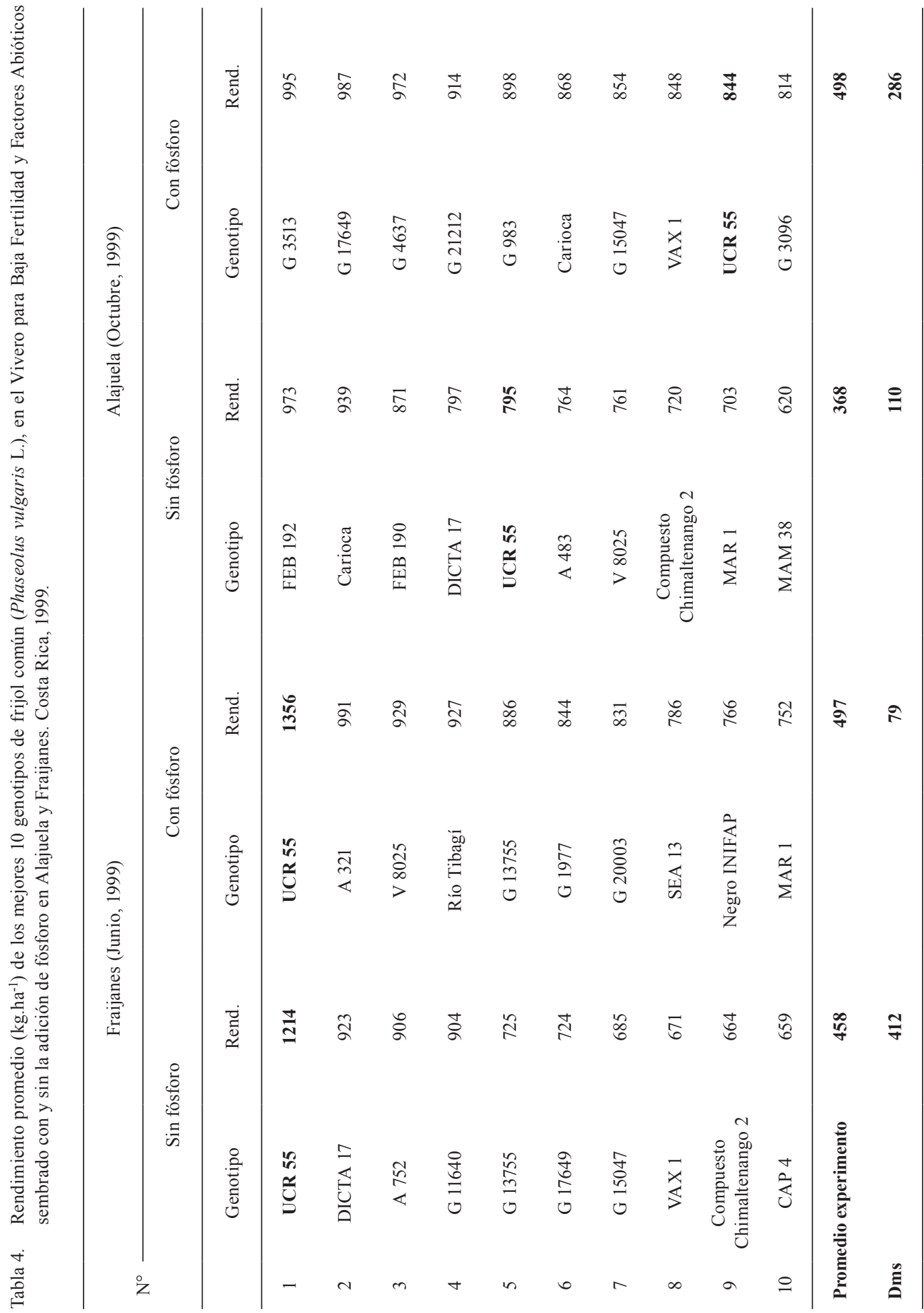


Durante los ENAR realizados en El Estero, el Vivero Histórico y Vivero LINAF en Alajuela, UCR 55 mostró una alta resistencia a C. lindemuthianum (antracnosis), y su calificación, excepto en el Vivero Histórico de 1999, fue igual o menor 3 (Tabla 5). La resistencia a ese patógeno fue evidente en el Vivero para Baja Fertilidad y Factores Abióticos, sembrado Fraijanes en 1999 (Figura 1). La antracnosis es una enfermedad común en zonas de altitud media y alta (sobre 1000 msnm) y con temperaturas frescas, de 18 a $22^{\circ} \mathrm{C}$ (Araya-Fernández 2009), por lo que UCR 55 se convirtió en una opción para áreas de producción con estas condiciones, usualmente situadas en la región Central de Costa Rica. Esta característica de la variedad, más su buen desempeño en condiciones de bajo fósforo, lograron explicar, los mayores rendimientos de UCR 55 en localidades alrededor o sobre los $1000 \mathrm{~m}$ de altura, como las de la zona de Puriscal, Alajuela y Fraijanes. 
Tabla 5. Reacción de la variedad de frijol común UCR 55 (Phaseolus vulgaris L.) a Colletotrichum lindemuthianum (antracnosis), Pseudocercospora griseola (mancha angular) y Thanatephorus cucumeris (mustia hilachosa) en el Ensayo Nacional de Adaptación y Rendimiento (ENAR), el Vivero Histórico de Frijol Común y el Vivero de Líneas Avanzadas de Frijol. Costa Rica. 1997-1999.

\begin{tabular}{|c|c|c|c|c|c|c|}
\hline \multirow{2}{*}{ Ensayo $^{\dagger}$} & \multirow{2}{*}{ Localidad } & \multirow{2}{*}{$\begin{array}{c}\text { Época } \\
\text { de siembra }\end{array}$} & \multicolumn{4}{|c|}{ Valor máximo ${ }^{\dagger \dagger}$} \\
\hline & & & UCR 55 & Guaymí & $\begin{array}{c}\text { Chirripó } \\
\text { Rojo }\end{array}$ & Bribrí \\
\hline \multicolumn{7}{|c|}{ Colletotrichum lindemuthianum (antracnosis) } \\
\hline ENAR & El Estero & 1997-B & 2 & 4 & 4 & 4 \\
\hline ENAR & El Estero & 1998-B & 2 & 2 & 4 & 3 \\
\hline Vivero histórico & Alajuela & 1998-A & 1 & 8 & 5 & 1 \\
\hline Vivero histórico & Alajuela & 1999-B & 4 & 7 & 6 & 5 \\
\hline Vivero LINAF & Alajuela & 1999-B & 3 & - & - & 4 \\
\hline \multicolumn{7}{|c|}{ Pseudocercospora griseola (mancha angular) } \\
\hline ENAR & Bijagual & 1997-B & 3 & 4 & 3 & 4 \\
\hline ENAR & La Legua & 1997-A & 2 & 2 & 2 & 4 \\
\hline ENAR & El Estero & 1997-B & 5 & 4 & 6 & 4 \\
\hline ENAR & El Estero & 1998-A & 3 & 4 & 6 & 5 \\
\hline Vivero histórico & Alajuela & 1998-A & 1 & 5 & 6 & 6 \\
\hline Vivero histórico & Alajuela & 1999-B & 4 & 5 & 4 & 4 \\
\hline Vivero LINAF & Alajuela & 1999-B & 3 & - & - & 5 \\
\hline \multicolumn{7}{|c|}{ Thanatephorus cucumeris (mustia hilachosa) } \\
\hline ENAR & Bijagual & 1997-B & 4 & 4 & 4 & 4 \\
\hline ENAR & Chánguena & 1997-B & 6 & 7 & 7 & 6 \\
\hline ENAR & Veracruz & 1998-A & 6 & 5 & 8 & 3 \\
\hline ENAR & El Estero & 1998-A & 4 & 4 & 5 & 4 \\
\hline ENAR & Bijagual & 1998-B & 6 & 6 & 7 & 6 \\
\hline ENAR & El Parque & 1998-C & 2 & 3 & 7 & 4 \\
\hline
\end{tabular}

$\dagger \quad$ Ensayo Nacional de Adaptación y Rendimiento (ENAR), Vivero Histórico de Frijol Común (Vivero histórico), Vivero de Líneas Avanzadas de Frijol (Vivero LINAF).

† Época de siembra = A (May-Ago); B (Oct-Ene); C (Dic-Mar).

行 Calificación de la enfermedad (escala de 1-9) donde $1=\sin$ síntomas visibles y 9 = síntomas de la efermedad muy severos (CIAT 1987).

Agronomía Costarricense 46(1): 77-94. ISSN:0377-9424 / 2022 


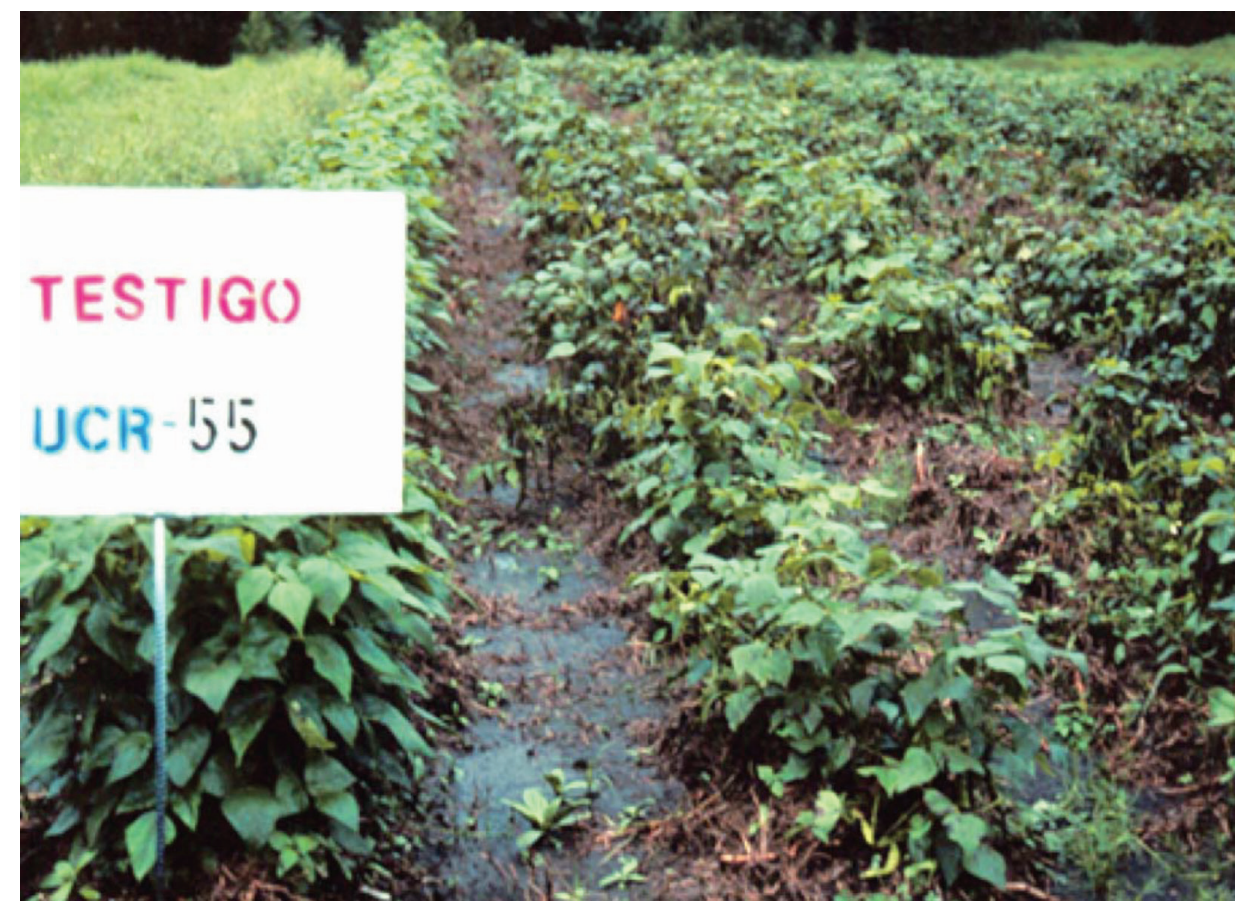

Figura 1. Reacción de la variedad UCR 55 (Phaseolus vulgaris L.) a la inoculación de conidios de Colletotrichum lindemuthianum (antracnosis) en el vivero para baja fertilidad y factores abióticos. Fraijanes, Costa Rica. 1999.

Adicionalmente, UCR 55 presentó resistencia intermedia a Pseudocercospora griseola y Thanatephorus cucumeris, con calificaciones menores a 6 en varios experimentos. En general, UCR 55 mostró igual o menor susceptibilidad que los testigos comerciales Guaymí y Chirripó Rojo, frente a estos 2 patógenos fungosos, que son los más comunes y responsables de pérdidas en rendimiento en las principales regiones productoras de Costa Rica. Al respecto, en estudios realizados en las localidades de Isla y Medellín de Bravo, Veracruz, y Tapachula, Chiapas, en México, UCR 55 se evidencia que mostró resistencia intermedia a $P$. griseola y a $C$. lindemuthianum con una calificación de 4 y resistencia a Uromyces appendiculatus (Pers.) Unger var. appendiculatus (roya) con una calificación de 2 (López-Salinas et al.
2006). El grado de resistencia a C. lindemuthianum, P. griseola, y T. cucumeris mostrado por UCR 55 , constata que bajo condiciones climáticas normales y buenas prácticas de manejo, que incluyen el uso de semilla de calidad, mínima labranza y un uso racional de fungicidas (Chaves-Barrantes y Araya-Fernández 2014), los patógenos fungosos no deberían constituir una limitante para la obtención de buenos rendimientos con esta variedad.

El rendimiento promedio de UCR 55 durante la fase de validación, bajo el manejo del productor, fue de $1336 \mathrm{~kg} . \mathrm{ha}^{-1}$, y varió desde 620 hasta 2345 kg.ha-1 (Tabla 6). Además, mostró poca adaptación en las localidades de altitudes menores a $750 \mathrm{msnm}$ (Concepción, El Parque y Veracruz), donde su rendimiento fue igual o menor al testigo de grano negro Guaymí y a la 
variedad de grano rojo Bribrí, reconocida por su buen rendimiento en las condiciones de esas comunidades. Sin embargo, se evidenció que el rendimiento de UCR 55, en localidades con una altitud superior a los $1000 \mathrm{msnm}$, como El Estero y Fraijanes, duplicó al de Guaymí y al de Bribrí, indicativo de su buena adaptación a altitudes medias o altas, y a suelos con baja disponibilidad de fósforo. El buen rendimiento de UCR 55 en la zona de Puriscal durante 1999, se ratificó en la prueba comercial llevada a cabo en el 2000 en los cantones de Puriscal, Mora y Aserrí, donde obtuvo una producción de $883 \mathrm{~kg} \cdot \mathrm{ha}^{-1}$ en $\mathrm{La}$ Legua, 1175 kg.ha- ${ }^{-1}$ en Cedral, 1867 kg.ha- ${ }^{-1}$ en Bajo Loaiza, 2162 kg.ha- ${ }^{-1}$ en El Estero y 2200 kg.ha- ${ }^{-1}$ en La Fila.

Tabla 6. Rendimiento promedio de la variedad de frijol común UCR 55 (Phaseolus vulgaris L.) en parcelas de validación en fincas de productores y bajo manejo comercial. Costa Rica, 1999-2000.

\begin{tabular}{|c|c|c|c|c|c|}
\hline \multirow{2}{*}{ Localidad } & \multirow{2}{*}{$\begin{array}{c}\text { Época } \\
\text { de siembra }{ }^{\dagger}\end{array}$} & \multicolumn{4}{|c|}{ Rendimiento promedio $\left(\mathrm{kg} \cdot \mathrm{ha}^{-1}\right)$} \\
\hline & & UCR 55 & Guaymí & Chirripó Rojo & Bribrí \\
\hline Concepción & 1999-A & 620 & 1442 & 1244 & 900 \\
\hline El Estero & 1999-A & 1345 & 655 & 791 & 677 \\
\hline Veracruz & 1999-A & 824 & 774 & 322 & 934 \\
\hline El Estero & 1999-B & 2162 & 706 & 399 & 746 \\
\hline Concepción & 1999-B & 1016 & 1114 & 764 & 695 \\
\hline Fraijanes & 1999-B & 2345 & 988 & 941 & 1310 \\
\hline El Parque & 1999-C & 1040 & 1318 & 941 & 1310 \\
\hline Media general & & 1336 & 1000 & 772 & 939 \\
\hline Dms & & & & & 472 \\
\hline
\end{tabular}

† Época de siembra = A (May-Ago); B (Oct-Ene); C (Dic-Mar).

Uno de los parámetros de calidad más importantes del grano de frijol es su tiempo de cocción, y es un factor fundamental para la aceptación de una variedad por los consumidores e industriales (valor comercial), ya que representa economía en el tiempo de preparación y en la energía utilizada (Mora 1982, Pérez-Herrera et al. 2002, Corrêa et al. 2010). Al respecto, la variedad UCR 55 mantuvo un tiempo de cocción menor a 80 min durante 150 días en condiciones de temperatura ambiente en Alajuela, en un experimento que evaluó el efecto del tiempo de almacenamiento sobre la calidad del grano (Oreamuno-Fonseca 2015). UCR 55 logró mantener su grano dentro de la categoría de primera calidad durante un tiempo aproximado de 5 meses, según el Reglamento Técnico RTCR 384:2004 Frijol en Grano (MEIC et al. 2005).

Con base en las condiciones en que fue evaluada y seleccionada, la variedad UCR 55 presentó un hábito de crecimiento indeterminado arbustivo (tipo II) y una arquitectura erecta y compacta. En altitudes iguales o menores a los 1100 msnm, alcanzó la floración entre los 33 y 36 días después de la siembra (dds), la madurez fisiológica entre los 65 y 70 dds, y la madurez de cosecha entre los 78 y 80 dds. En altitudes superiores a los $1700 \mathrm{msnm}$ la floración se alcanzó entre los 55 y 60 dds, la madurez fisiológica entre los 91 y 99 dds, y la madurez de cosecha entre los 104 y 112 dds; debido a que la velocidad del crecimiento y desarrollo de las plantas está en función de la temperatura (Salysbury y Ross 1994, Hatfield y 
Prueger 2015). Las flores de UCR 55 son moradas (Figura 2) y las vainas inmaduras verdes; además, al iniciar la maduración, las vainas adquirieron un jaspe morado en la sutura dorsal y al completarse, se tornan amarillo claro con jaspes morados (Figura 3). En ocasiones también presentan

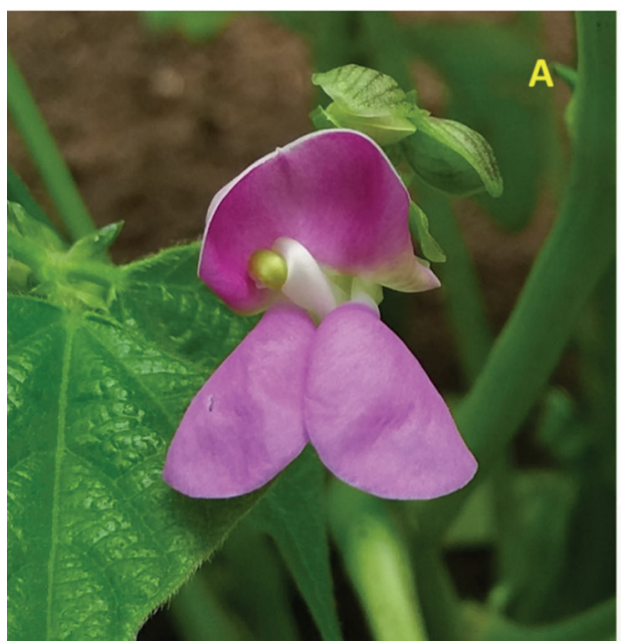

estrías moradas. Las vainas secas son de color crema (Figura 3). Adicionalmente se identificó que el promedio de semillas por vaina es de 6 , con forma ovoide y color negro opaco al secarse (Figura 2). El peso promedio de 100 semillas fue de $19,4 \mathrm{~g}$ (al 14\% de humedad).

Figura 2. Variedad UCR 55 (Phaseolus vulgaris L.). (A) Flor, (B) Semillas, Costa Rica, 2021.

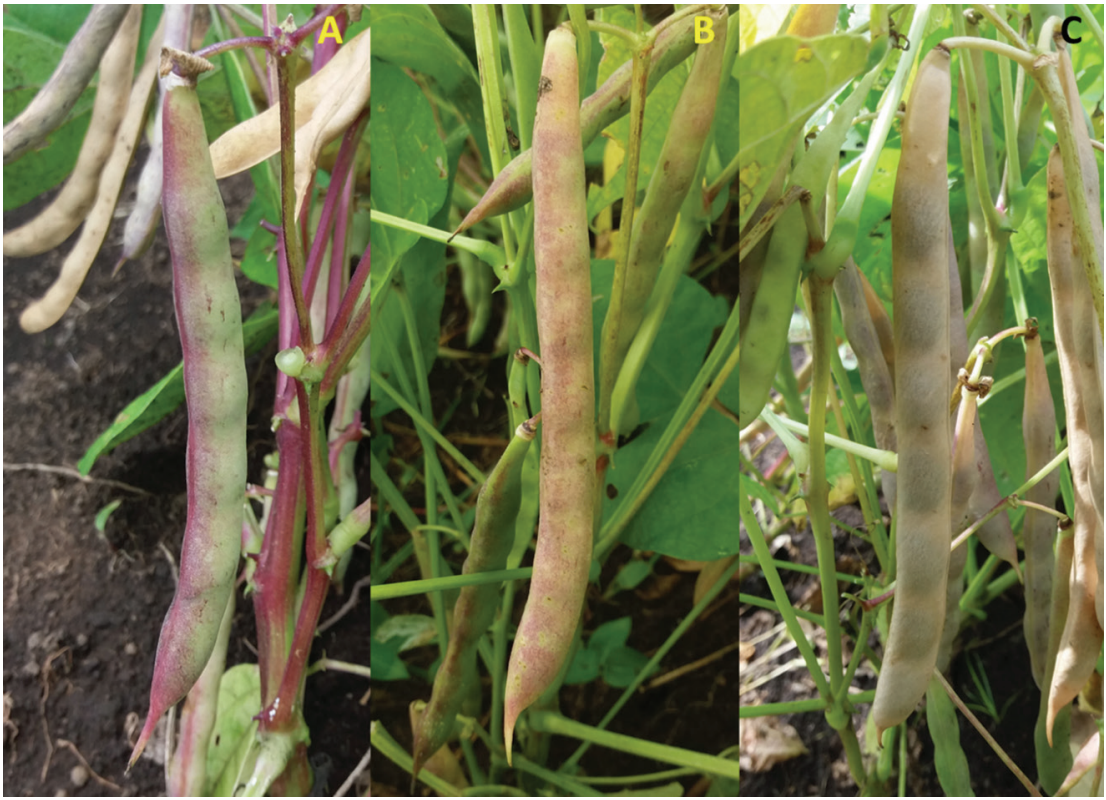

Figura 3. Variedad UCR 55 (Phaseolus vulgaris L.). (A) Vainas en inicio de madurez fisiológica, (B) Al completar la madurez, (C) Punto de cosecha. Costa Rica, 2021. 
A pesar de que la evaluación de la variedad UCR 55 finalizó en el 2000 con las pruebas comerciales, y ya han pasado más de 20 años desde las investigaciones, se identificó como el único cultivar con buena adaptación a suelos con bajo $\mathrm{P}$ y resistencia a $C$. lindemuthianum del que disponen los productores costarricenses. Las demás variedades de grano negro que se utilizan en la actualidad, como Brunca, Guaymí, Matambú y Nambí no fueron generadas para enfrentar esta limitante edáfica.

Semilla de la variedad UCR 55 puede ser obtenida en la Estación Experimental Agrícola Fabio Baudrit Moreno (EEAFBM) de la UCR.

\section{RECONOCIMIENTO}

La variedad UCR 55 es producto del trabajo conjunto entre la Universidad de Costa Rica (UCR) y el Ministerio de Agricultura y Ganadería (MAG) de Costa Rica, en colaboración con Programa de Frijol del Centro Internacional de Agricultura Tropical (CIAT) en Colombia.

\section{AGRADECIMIENTOS}

La investigación que dio origen a UCR 55 fue financiada por la Universidad de Costa Rica (proyecto 736-91-315, inscrito en la Vicerrectoría de Investigación), y por el Ministerio de Agricultura y Ganadería (MAG) de Costa Rica. También recibió financiamiento parcial del Programa Cooperativo Regional de Frijol de Centroamérica, México y El Caribe (PROFRIJOL). Se agradece el apoyo del PhD. Stephen E. Beebe del Programa de Frijol del Centro Internacional de Agricultura Tropical (CIAT), en la generación de la variedad UCR 55.

\section{LITERATURA CITADA}

Alvarado, A; Bertsch, F; Bornemisza, E; Cabalceta, G; Forsythe, W; Henríquez, C; Mata, R; Molina, E; Salas, R. 2001. Suelos derivados de cenizas volcánica (Andisoles) de Costa Rica. San José, Costa Rica, Asociación Costarricense de la Ciencia del Suelo. 111 p.
Araya-Fernández, CM. 2009. Enfermedades y su combate. In Cultivo de frijol (Phaseolus vulgaris). Manual de recomendaciones técnicas para el cultivo de frijol. Hernández, JC; Ramírez, L (eds.). San José, Costa Rica, Instituto Nacional de Innovación y Transferencia en Tecnología Agropecuaria (INTA). p. 63-72.

Araya, C; Hernández, JC. 2003. Distribución agroecológica de enfermedades del frijol en Costa Rica. Manejo Integrado de Plagas y Agroecología 68:26-33.

Araya, R; Zamora, A. 1988. Efecto del frijol común sobre la productividad del cafeto podado y en formación. Agronomía Costarricense 13(1):93-97.

Assefa, T; Assibi-Mahama, A; Brown, AV; Cannon, EKS; Rubyogo, JC; Rao, IM; Blair, MW; Cannon, SB. 2019. A review of breeding objectives, genomic resources, and marker-assisted methods in common bean (Phaseolus vulgaris L.). Molecular Breeding 39:20. DOI: 10.1007/s11032-018-0920-0.

Beebe, SE. 2012. Common bean breeding in the tropics. Plant Breeding Reviews 36(1):357-426.

Beebe, S; Rao, I; Blair, MW; Butare, L. 2009. Breeding for abiotic stress tolerance in common bean: present and future challenges. Paper presented at: 14th Australian Plant Breeding and 11th Society for Advancement of Breeding Research in Asia and Oceania (SABRAO), Brisbane, Australia. 10-14 Aug. s.p.

Beebe, SE; Rojas-Pierce, M; Yan, X; Blair, MW; Pedraza, F; Muñoz, F; Tohme, J; Lynch, JP. 2006. Quantitative trait loci for root architecture traits correlated with phosphorus acquisition in common bean. Crop Science 46:413-423. DOI: 10.2135/cropsci2005.0226.

Camilo, S; Odindo, AO; Kondwakwenda, A; Sibiya, J. 2021. Root traits related with drought and phosphorus tolerance in common bean (Phaseolus vulgaris L.). Agronomy 11, 552. DOI: 10.3390/ agronomy11030552.

Chaves-Barrantes, NF; Araya-Fernández, CM. 2012. Pérdidas causadas por el amachamiento del frijol (Aphelenchoides besseyi Christie) y reacción del germoplasma comercial al patógeno. Agronomía Mesoamericana 23(1):01-12. DOI: 10.15517/ AM.V23I1.2013.

Chaves-Barrantes, NF; Araya-Fernández, CM. 2014. Buenas Prácticas Agrícolas (BPA) en el Cultivo de Frijol. Heredia, Costa Rica, Editorial de la Universidad Nacional (EUNA). 129 p.

Chaves, N; Cervantes, E; Zabalgogeazcoa, I; Araya, CM. 2013. Aphelenchoides besseyi Christie (Nematoda: Aphelenchoididae), agente causal del amachamiento del frijol común. Tropical Plant Pathology 38(3):243-252.

CIAT (Centro Internacional de Agricultura Tropical). 1987. Sistema estándar para la evaluación de germoplasma 
de frijol. In Von Schoonhoven, A; Pastor-Corrales, M (eds.). Cali, Colombia, CIAT. 56 p.

Corrêa, MM; De Carvalho, LMJ; Nutti, MR; De Carvalho, JLV; Neto, ARH; Ribeiro, EMG. 2010. Water absorption, hard shell and cooking time of common beans (Phaseolus vulgaris L.). African Journal of Food Science and Technology 1:13-20.

González, W; Gutiérrez, R; Araya, R. 1986. Análisis económico de la asociación cafeto (Coffea arábica L.) frijol (Phaseolus vulgaris L.) en cafetales con poda sistémica. Boletín Técnico Estación Experimental Fabio Baudrit M. 19(3):1-9.

Hatfield, JL; Prueger, JH. 2015. Temperature extremes: Effect on plant growth and development. Weather and Climate Extremes 10(A):4-10. DOI: $10.1016 / \mathrm{j}$. wace. 2015.08.001

Hernández-Fonseca, JC. 2009a. Clima y suelos. In Cultivo de frijol (Phaseolus vulgaris). Manual de recomendaciones técnicas para el cultivo de frijol. Hernández, JC; Ramírez, L (eds.). San José, Costa Rica, Instituto Nacional de Innovación y Transferencia en Tecnología Agropecuaria (INTA). p. 17.

Hernández-Fonseca, JC. 2009b. Zonas de cultivo y épocas de siembra. In Cultivo de frijol (Phaseolus vulgaris). Manual de recomendaciones técnicas para el cultivo de frijol. Hernández, JC; Ramírez, L (eds.). San José, Costa Rica, Instituto Nacional de Innovación y Transferencia en Tecnología Agropecuaria (INTA). p. 18.

Hernández-Fonseca, JC; Araya-Villalobos, R. 2009. Cultivo. In Cultivo de frijol (Phaseolus vulgaris). Manual de recomendaciones técnicas para el cultivo de frijol. Hernández, JC; Ramírez, L (eds.). San José, Costa Rica, Instituto Nacional de Innovación y Transferencia en Tecnología Agropecuaria (INTA). p. 19-26.

Hernández, JC; Araya, R; González, W. 1999. Diagnóstico de la actividad productiva del frijol (Phaseolus vulgaris L.) en la región Brunca de Costa Rica: cosecha 1995-1996. Agronomía Mesoamericana 10(2):75-84. DOI: 10.15517/AM.V10I2.17946.

Hernández, JC; Araya, R; Morales, A. 2001. Bribrí, nueva variedad de frijol rojo pequeño para Costa Rica. Agronomía Mesoamericana 12(1):15-23. DOI: 10.15517/AM.V12I1.17242.

Holdridge, LR. 1978. Ecología basada en zonas de vida. San José, Costa Rica, IICA. 216 p.

Ishimoto, M; Chrispeels, MJ. 1996. Protective mechanism of the Mexican bean weevil against high levels of a-amylase inhibitor in the common bean. Plant Physiology 111:393-401.

López-Salinas, E; Tosquy-Valley, OH; Villar-Sánchez, B; Becerra-Leor, EN; Ugalde-Acosta, FJ; CumpiánGutiérrez, J. 2006. Adaptabilidad de genotipos de frijol resistentes a enfermedades y suelos ácidos. Revista de Fitotecnia Mexicana 29(1):33-39.

Lynch, JP. 2019. Root phenotypes for improved nutrient capture: An underexploited opportunity for global agriculture. New Phytologist 223(2):548-564. DOI: 10.1111/nph.15738.

Miklas, PN; Kelly, JD; Beebe, SE; Blair, MW. 2006. Common bean breeding for resistance against biotic and abiotic stresses: From classical to MAS breeding. Euphytica 147:105-131. DOI: 10.1007/ s10681-006-4600-5.

MEIC (Ministerio de Economía, Industria y Comercio), Ministerio de Salud, MAG (Ministerio de Agricultura y Ganadería). 2005. Decreto Ejecutivo $\mathrm{N}^{\circ}$ 32149-MEIC-S-MAG. Reglamento Técnico RTCR 384:2004 Frijol en Grano. Diario oficial La Gaceta N ${ }^{\circ} .3$ del 05 de enero del 2005.

Mora, M. 1982. Influencia de diferentes temperaturas y contenidos de humedad sobre el tiempo de cocción de frijol (Phaseolus vulgaris L.) almacenado durante 18 meses. Agronomía Costarricense 6(1/2):87-89.

Mourice, SK; Tryphone, GM. 2012. Evaluation of common bean (Phaseolus vulgaris L.) genotypes for adaptation to low phosphorus. International Scholarly Research Network Agronomy, 309614:19. DOI:10.5402/2012/309614.

Muñoz, G; Giraldo, G; De Soto, JF. 1993. Descriptores varietales: arroz, frijol, maíz, sorgo. Cali, Colombia, Centro Internacional de Agricultura Tropical (CIAT). 174 p.

Oreamuno-Fonseca, P. 2015. Efecto de las altas temperaturas en el campo, el momento de cosecha y el almacenamiento tradicional sobre el tiempo de cocción y la imbibición de los granos de variedades de frijol común. Tesis MSc. San Pedro, Costa Rica, Universidad de Costa Rica. 108 p.

Pérez-Herrera, P; Esquivel-Esquivel, G; Rosales-Serna, R; Acosta-Gallegos, JA. 2002. Caracterización física, culinaria y nutricional de frijol del altiplano subhúmedo de México. Archivos Latinoamericanos de Nutrición 52:172-180.

Ramaekers, L; Remans, R; Rao, IM; Blair, MW; Vanderleyden, J. 2010. Strategies for improving phosphorus acquisition efficiency of crop plants. Field Crops Research 117:169-176.

Rodríguez-González, S; Fernández-Rojas, SE. 2015. Prácticas culinarias asociadas al consumo de frijoles en familias costarricenses. Agronomía Mesoamericana 26(1):145-151. DOI: 10.15517/ am.v26i1.16937.

Rosas, JC; Castro, A; Flores, E. 2000. Mejoramiento genético del frijol rojo y negro mesoamericano para Centroamérica y el Caribe. Agronomía Mesoamericana 11(2):37-46. DOI: $10.15517 /$ AM.V11I2.17305. 
Rosas, JC; Guachambala, M; Ramos, RA. 2009. Guía ilustrada para la descripción de las características de variedades del frijol común. Honduras, Escuela Agrícola Panamericana, Zamorano. 22 p.

Rosas, JC; Hernández, JC; Araya, R. 2003. Registration of 'Bribri' Small Red Bean (Race Mesoamerica). Crop Science 43(1):430-431.

Salysbury, FB; Ross, CW. 1994. Fisiología vegetal. México, Grupo Editorial Iberoamérica. 759 p.

Singh, SP; Schwartz, HF. 2010. Breeding common bean for resistance to diseases: a review. Crop Science 50:2199-2223.

Singh, SP; Schwartz, HF. 2011. Breeding common bean for resistance to insect pest and nematodes. Canadian
Journal of Plant Science 91(2):239-250. DOI:10.4141/ CJPS10002

Singh, SP; Terán, H; Muñoz, CG; Osorno, JM; Takegami, JC; Thung, MD. 2003. Low soil fertility tolerance in landraces and improved common bean genotypes. Crop Science 43:110-119. DOI:10.2135/ cropsci2003.0110.

Smith, MR; Veneklaas, E; Polania, J; Rao, IM; Beebe, SE; Merchant, A. 2019. Field drought conditions impact yield but not nutritional quality of the seed in common bean (Phaseolus vulgaris L.) PLoS ONE 14(6):e0217099. DOI:10.1371/journal. pone.0217099. 